\title{
ENHANCED PERFORMANCE OF THE ADVANCED LIGHT SOURCE THROUGH PERIODICITY RESTORATION OF THE LINEAR LATTICE ${ }^{*}$
}

\author{
D. Robin, C. Steier, LBNL, Berkeley, CA, USA \\ J. Safranek, SSRL, Stanford, CA, USA \\ W. Decking, DESY, Hamburg, Germany
}

\begin{abstract}
An essential feature of third generation storage ring based light sources is the magnetic lattice is designed with a high degree of periodicity. Tracking simulations show that if the periodicity is perturbed (by focusing errors for example), non-linear resonances become excited, which causes a reduction in the dynamic aperture. Therefore it is important to have a method to measure and correct perturbed periodicity. In this paper we study the effect of broken and restored periodicity at an actual third generation light source: the Advanced Light Source (ALS) at Lawrence Berkeley National Laboratory. First we show that it is possible to accurately determine the storage ring optic and thus the perturbation of the periodicity by fitting measured orbit response matrices. This method allows us to determine individual field gradient errors in quadrupoles and closed orbit errors in sextupoles. By varying individual quadrupole field strengths it is possible to correct the optic, largely restoring the lattice periodicity. A comparison is made of the performance of the ALS before and after the optic is corrected. Measurements of the electron beam tails and the synchrotron light image reveal a large suppression in resonance excitation after the optic is corrected. Correcting the optic also improves the injection efficiency and lifetime.
\end{abstract}

\section{INTRODUCTION}

The performance of the ALS storage ring is determined to a large degree by the single particle dynamics and nonlinear resonance excitation. Nonlinear resonance excitation can lead to many undesirable effects:

1. Small dynamic aperture

2. Short lifetimes (intrabeam and gas-scattering)

3. Slow injection rates

4. Distortion in the beam shape

5. Reduction in the betatron tune space where the machine can operate.

A resonance occurs when there is an integer relation between the horizontal and vertical betatron tunes $\nu_{x}, \nu_{y}$, and

* This work was supported by the Director, Office of Energy Research, Office of Basic Energy Sciences, Materials Sciences Division of the U.S. Department of Energy, under Contract No. DE-AC03-76SF00098. the longitudinal revolution frequency $\nu$, which is normalized to $\nu=1$, i.e.

$$
N_{x} \nu_{x}+N_{y} \nu_{y}+R=0
$$

where $N_{x}, N_{y}$, and $R$ are integers. If the lattice is $M$-fold periodic, its dynamics is the same as the dynamics of a single sector with longitudinal frequency $\nu^{\prime}=M$. A resonance will occur only when $R=R^{\prime} \times M$, that is when $R$ is evenly divisible by M. The ALS magnetic lattice is constructed of twelve identical sectors. So the ALS's 12-fold periodicity is beneficial in suppressing many resonances.

However these resonances that were suppressed by periodicity become excited when focusing errors, such as quadrupole field errors and orbit errors in sextupoles, perturb the periodicity of the ring. The strength of resonance excitation depends on the degree of periodicity breaking. Through analysis of measured orbit response matricies we have measured and reduced the perturbation of the periodicity. This has yielded significant improvements in the machine performance. In this paper we show how the understanding and performance of the storage ring has been enhanced by restoring the linear lattice periodicity.

\section{DETERMINING THE LINEAR LATTICE}

The method used to determine the magnet gradient distribution in the ALS is by analyzing measured orbit response matrix data. This analysis method has been suggested by Corbett, Lee and Ziemann at SLAC [1] and refined by Safranek at BNL $[2,3]$. The first analysis of ALS orbit response matrices was made in 1994 shortly after commissioning by Bengtsson and Meddahi [4]. More extensive analysis was done by Robin et al. [5, 6] for the uncoupled linear lattice and more recently extended to the coupled linear lattice by Steier and Robin. [7].

An orbit response matrix, $\mathbf{M}$, is defined by

$$
\left(\begin{array}{l}
\vec{x} \\
\vec{y}
\end{array}\right)=\mathrm{M}\left(\begin{array}{c}
\vec{\theta}_{\mathrm{x}} \\
\vec{\theta}_{\mathrm{y}}
\end{array}\right)
$$

where $\overrightarrow{\theta_{x}}, \overrightarrow{\theta_{y}}$ are changes in steering magnet strengths and $\vec{x}, \vec{y}$ are the resulting electron orbit perturbations at the beam position monitors (BPMs). The measured orbit response matrix, $\mathbf{M}_{\text {meas }}$, is obtained by changing a steering magnet and measuring orbit changes with BPMs. Also it is possible to calculate the orbit response matrix, $\mathbf{M}_{\text {mod }}$, 
assuming some gradient distribution in the storage ring and using an optic model. If the assumed gradient distribution is wrong then $\mathbf{M}_{\text {meas }}$ and $\mathbf{M}_{\text {mod }}$ will not be the same. The actual gradient distribution in the ring is then determined by adjusting the gradient distribution in the model to minimize the difference between $\mathbf{M}_{\text {meas }}$ and $\mathbf{M}_{\text {mod }}$. In the ALS there are 31488 elements in the fully coupled matrix and 15744 elements in the uncoupled matrix. For the analysis we used the optics codes COMFORT [8] and TRACYII [9]. The parameters in the COMFORT and TRACY-II models were varied to minimize the $\chi^{2}$ deviation between the model and measured orbit response matrices using the computer code LOCO [3].

Because it is possible at the ALS to store beam with the sextupoles turned off the model calibration is done in two steps. First a response matrix is measured and fitted with the sextupoles turned off. We use 50 gradient parameters in the fit - one associated with each quadrupole power supply. Then a response matrix is measured and calibrated with the sextupoles turned on. Keeping the initial 50 gradient parameters fixed we fit 48 new gradient parameters - one associated with each sextupole. In this manner we are able to better isolate the linear focussing errors of the quadrupoles from orbit errors in the sextupoles. For more details about the ALS response matrix fit we refer the reader to [6].

\subsection{Quadrupole Field Errors}

Initial LOCO analyses of the ALS storage ring were made in the fall of 1995. The fits revealed that of the two independently powered quadrupole families, the $Q D$ family had significantly larger variation in field than the $Q F$ family. In particular the rms variation in quadrupole field strengths within each quadrupole family was determined to be $0.20 \%$ for the QF quadrupoles and $0.63 \%$ for the $Q D$ quadrupoles. These $Q D$ values are particularly large when compared with the quadrupole design tolerances which specify that within a family the field variation should lie within a band of $\pm 0.2 \%$. Although the accuracy of the method is difficult to determine, there was only a $0.1 \%$ rms variation in the fit quadrupole gradients from data set to data set.

We assumed that any large variation in quadrupole fields is more likely a result of the variation in the power supply than in the mechanical construction. Independently each magnet power supply excitation current was measured using a hand held current monitor [10]. A magnet-to-magnet comparison was made between the quadrupole strengths obtained with the current meter and the response matrix fitting. The results are shown in Fig. 1. The rms difference between the two measurements is $0.2 \%$ and is within the precision of the current monitor $( \pm 0.3 \%)$. Since these two independent measurement methods produced consistent results, it gave us confidence that the two methods are capable of pinpointing individual magnet field strength errors to a precision better than $0.3 \%$. It was ultimately shown that the reason that power supplies were not regulating to

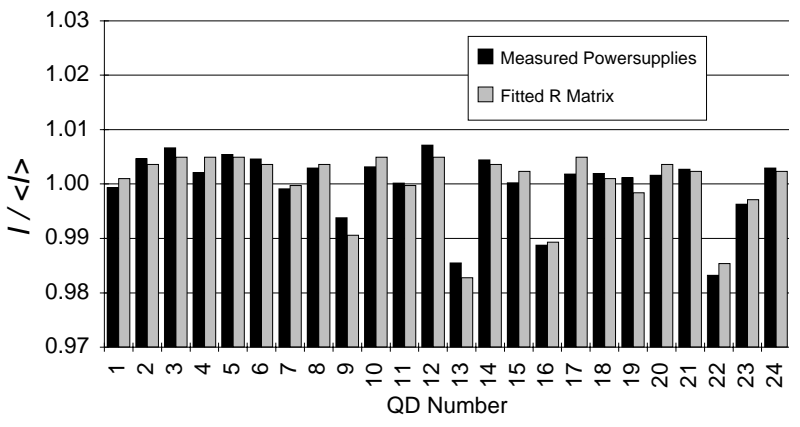

Figure 1: A comparison of the relative variation of $Q D$ quadrupole strengths as measured with a current monitor and fitted response matrix.

specification was due to malfunctioning regulating shunts.

One measure of the degree of periodicity breaking is the distortion of the $\beta$-function in the ring. Using the calibrated model it is possible to compute the $\beta$-function. The rms perturbation of the $\beta$-function from the ideal $\beta$-function is $3 \%$ horizontally and $15 \%$ vertically. The perturbation of the $\beta$-function is displayed in Fig. 2 (top).

\subsection{Orbit Errors in Sextupoles}

Horizontal closed orbit distortions, $x_{0}$, in a sextupole of strength $S$ feed down to a quadrupole component $K_{s}$ :

$$
K_{s}=2 S x_{0} .
$$

To determine $x_{0}$, the response matrix with sextupoles turned on is fitted keeping the quadrupole gradients fixed to the value determined previously with sextupoles turned off. From this fit we determine the $48 K_{s}$. Then the sextupole gradients, $S$, are determined by adjusting the sextupoles in the model to correct the linear chromaticity to the measured value. In our fits we assumed the same sextupole strength for all the $24 S F$ sextupoles and the same sextupole strength for all the $24 S D$ sextupoles. The assumption is made that there is little variation in $S$ among the sextupoles in a family because all the sextupoles in a family are powered by one supply. Having values of $K_{s}$ and $S$ for each of the sextupoles, equation 3 is used to determine the offsets, $x_{0}$.

The horizontal offsets in the sextupoles, which were as much as $1.5 \mathrm{~mm}$ in the $S F \mathrm{~s}$ and $0.8 \mathrm{~mm}$ in the $S D$ s, resulted in an additional increase in the amplitude of beating of the $\beta$-function. As can be seen in Fig. 2 (middle) the rms perturbation of the $\beta$-function is $6 \%$ horizontally and $19 \%$ vertically. Even though there is some increase in the distortion of the $\beta$-function due to the offsets most of the distortion was a result of the mispowered quadrupoles.

\subsection{Restoring the periodicity of the lattice}

Once the real lattice is known it is possible to adjust quadrupoles to correct the optic and restore the lattice periodicity. Choosing how the quadrupoles get set depends 

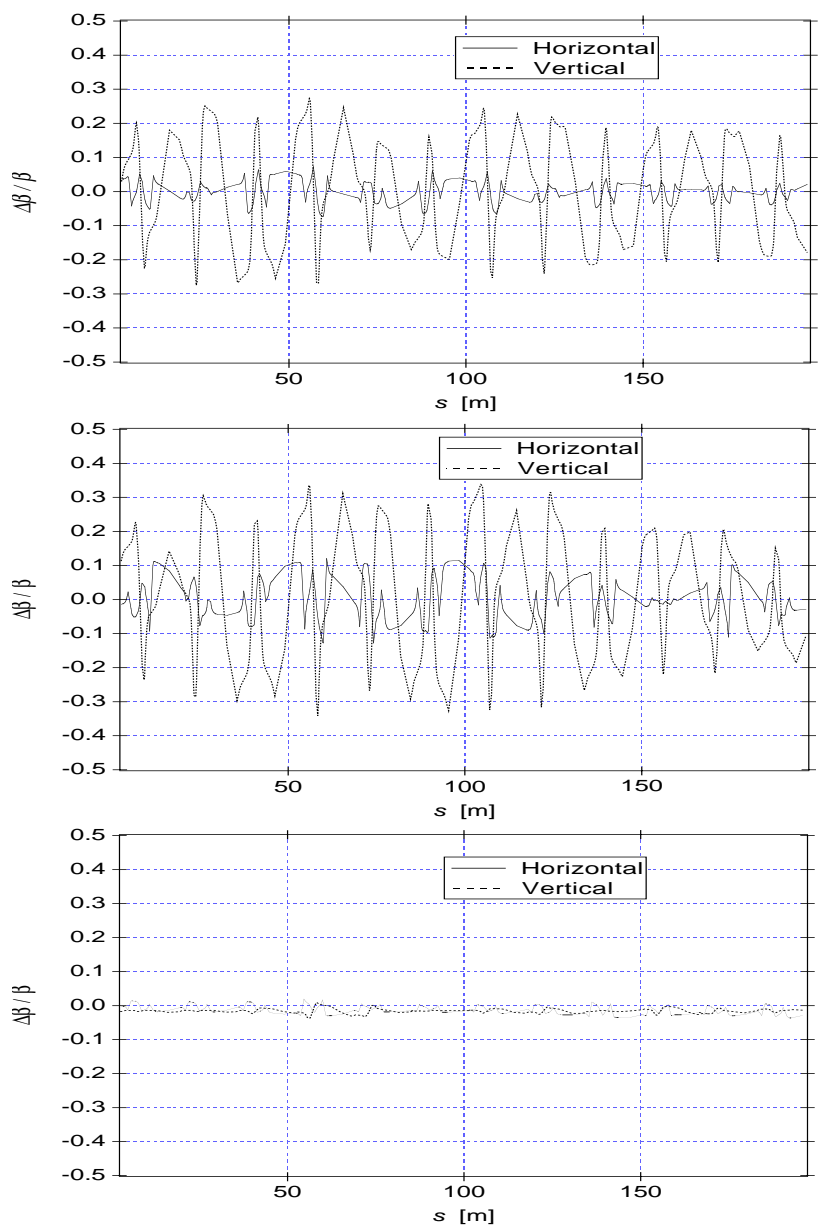

Figure 2: Horizontal and vertical $\beta$-beating with sextupoles turned off (top), with sextupoles turned on (middle), and with sextupoles turned on after the periodicity is corrected (bottom).

on what is important. In our case we chose the following merit function: Adjust the quadrupoles to minimize the difference between the actual and ideal response matrix. Because we have distributed gradient errors this merit function effectively corrects both the $\beta$-beating and the transfer matrices between sextupoles. Determining the quadrupole values that best correct the optic is also done with LOCO, and the algorithm is as follows. We have 49 individual parameters that can be adjusted to compensate the $\beta$-beating in the ring: each one of the $Q F$ and $Q D$ quadrupole magnets which can be adjusted individually and the $Q F A$ quadrupoles that can be adjusted as a group. We fit the orbit response matrix with sextupoles on keeping the gradients in the sextupoles zero in the fit model and varying only the 49 quadrupole gradient parameters. We then find the variation in those 49 parameters that best reproduces the break in periodicity of the measured response matrix. We then adjust the quadrupole power supplies in the ring with just the opposite variation. A new set of response matrices is measured and the model is refit. From this we compute the $\beta$-beating of the lattice again and find that it had reduced to less than 1\% RMS (see Fig. 2 (bottom)).

\section{EFFECT OF RESTORED PERIODICITY}

Once the periodicity was corrected we experienced a dramatic improvement in the performance of the storage ring. At a beam energy of $1.5 \mathrm{GeV}$ both the lifetime and injection rates were better than before the periodicity was restored. Before the periodicity was restored it was necessary to put in a DC orbit bump in the injection straight, moving the stored beam closer to the injection septum, in order to have a reasonable injection efficiency. After we restored the periodicity it was possible to have a large injection rate without the DC bump. We also experienced roughly a $20 \%$ increase in lifetime after the periodicity was restored. Both of these effects we think are due to the reduction in resonance excitation and the increase in the dynamic aperture. The reduction in resonance excitation is directly seen in several ways - beam tail measurements, beam profile measurements, and measured frequency maps.

\subsection{Beam tail measurements}

When excited, structural resonances may alter the behavior of particles in the beams tail. Resonances may cause particles to increase and decrease their transverse amplitudes or to be trapped at large amplitudes (for instance particles may get trapped in resonance islands). Therefore by monitoring changes in the beam tails as the betatron tunes are varied it is possible to observe the onset of resonances.

The way in which we monitor the tails is by limiting the transverse physical aperture with a beam scraper and measuring the beam lifetime as a function of the betatron tunes. If resonances are present in the vicinity of the tunes and there is a change in the beam shape, there will be a change in the number of particles that hit the scraper when they make large amplitude excursions resulting in a change in the beam lifetime. Thus if we vary the betatron tunes while simultaneously observing the beam lifetime we will see the lifetime drop or rise when we move onto excited resonances. The experimental technique is very similar to that which was used in VEPP-4 [11] to measure the effect of the beam-beam force on the tails of the beam. A beam loss monitor consisting of two plastic scintillators with photomultiplier tube's outputs in coincidence $(\gamma-$ telescope) was located just down-stream of a horizontal and vertical scraper [12]. The $\gamma$-telescope detects gamma radiation emitted when electrons hit the scraper. The count rate detected is proportional to the rate at which particles hit the scraper and is inversely proportional to the beam lifetime. Therefore by observing the change in the ratio of the beam current to the detector count rate as a function of betatron tune we can observe a change in lifetime and thus the onset of resonance excitation.

Our experimental procedure was the following. We would first change the tunes by changing two families of 
quadrupoles, $Q F$ and $Q D$, according to a previously measured tune sensitivity matrix. After the quadrupole fields have settled we measured the beam current and the count rate in the detector for a 1 second interval. Then move on to the next tune. In order to check how well the predicted tunes agree with the measured tunes we would periodically measure the tunes.

We chose to scan in a region of tune space where two resonances are present: $5 \nu_{x}=72$ (allowed by periodicity) and $3 \nu_{x}=43$ (unallowed by periodicity). Two scans were made before and after we corrected the optics. In Fig. 3 we plotted on the vertical axis is the count rate divided by current and on the bottom axis the horizontal tune. We scanned the horizontal tune from $14.3<\nu_{x}<14.45$ keeping the vertical tune constant at $\nu_{y}=8.15$. Looking at the figure one can see that the amplitude of the $5 \nu_{x}=72$ resonance remains constant whereas the amplitude of the $3 \nu_{x}=43$ has been greatly suppressed due to the periodicity restoration.

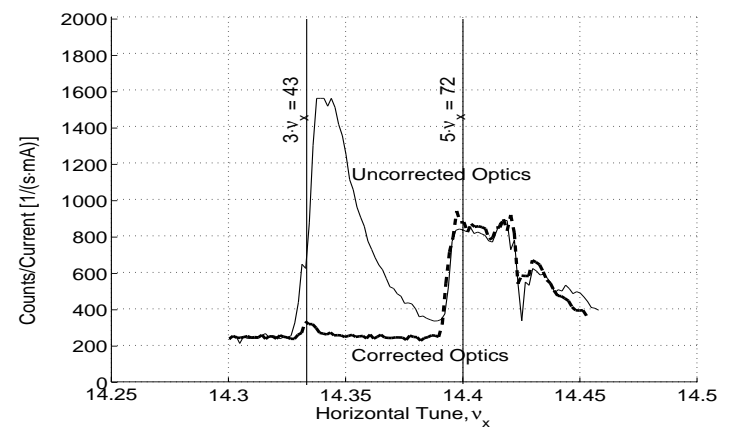

Figure 3: Horizontal tunescans before (thin,solid) and after (thick,dotted) the optic is corrected. The vertical tune is kept constant at 8.15. At each horizontal tune, the count rate (as measured in a gamma counter) divided by beam current is plotted.

The effects of periodicity breaking on the behavior of the tails at different tunes are illustrated with the help of phase space plots calcutated for the fitted lattices. Fig. 4 shows the horizontal phase spaces near to the $3 \nu_{x}=43$ resonance. The left side shows the phase space of the optic before periodicity was restored, the right side shows the phase space of the ideal optic.

In the vicinity of the $3 \nu_{x}$ resonance the inner phase space becomes completely distorted by the islands of the 3rd integer resonance when the periodicity is broken whereas in the unperturbed case there is no distortion at all.

We expect that with islands in the phase space particles can get launched and then captured in the tails either through gas scattering or intrabeam scattering. This distorts the distribution of the beam leading to an increase in the beam loss rate. From the phase space pictures in Fig. 4 we expect that the tail distributions and the count rate in
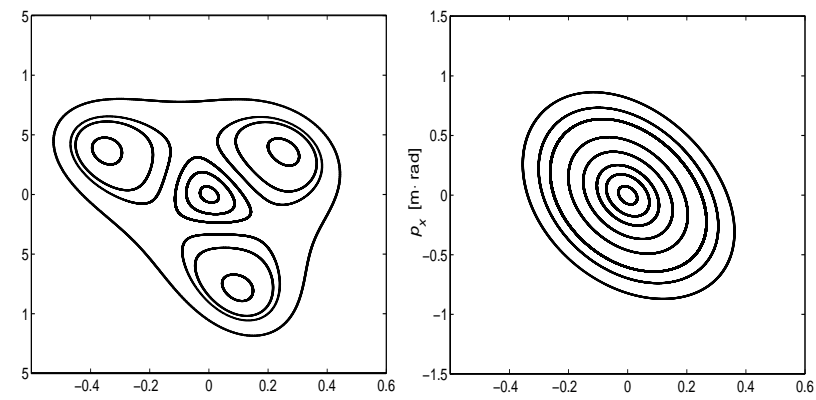

Figure 4: Horizontal phase space of the lattice plotted at the observation point. Lattices are tuned to $\nu_{x}=14.3338$ and $\nu_{y}=8.15$. Left plot shows the phase space with the uncorrected optic. Right plot shows the phase space for the corrected optic.

the vicinity of the $3 \nu_{x}$ resonance would be higher before the periodicity was restored. This is consistent with what we observed experimentally (see Fig. 3).

\subsection{Beam profile monitor}

We have the ability to observe the beam distribution with a beam profile monitor. This monitor images the synchrotron radiation that is emitted from a bending magnet and represents roughly a one-to-one image of the beam. An image of the beam near the $3 \nu_{x}$ resonance can be seen in Fig. 5 before (left) and after (right) the periodicity was restored. What we see is that in the lattice before the periodicity was restored the beam "splits" into several spots. However that was not the case after the periodicity was restored.

Again the phase space plot, Fig. 4, can be used to help us understand the image. The tracking point of the phase space corresponds to the observation point of the beam. One can imagine that if the islands are populated the projection onto the normal space would look something like the image. The reason that one sees only three spots and not four is that when one projects the phase space onto the $x$-axis two islands overlap. The phase space plot is only meant to serve as a qualitative understanding of the image. We make no quantitative statements about the distribution.

A more sensitive test of the resonance excitation can be made by experimentally measuring a frequency map using turn-by-turn magnetic kicker and a single turn beam position monitor. For details of the frequency map measurement the reader is referred to the following papers $[13,14]$.

\section{CONCLUSION}

The periodicity of the linear lattice is clearly important for the ALS. The actual linear lattice is accurately determined by analyzing orbit response matrices. From this analysis we have found power supply errors and orbit offsets in sextupoles which lead to a beating of the vertical $\beta$-function of 

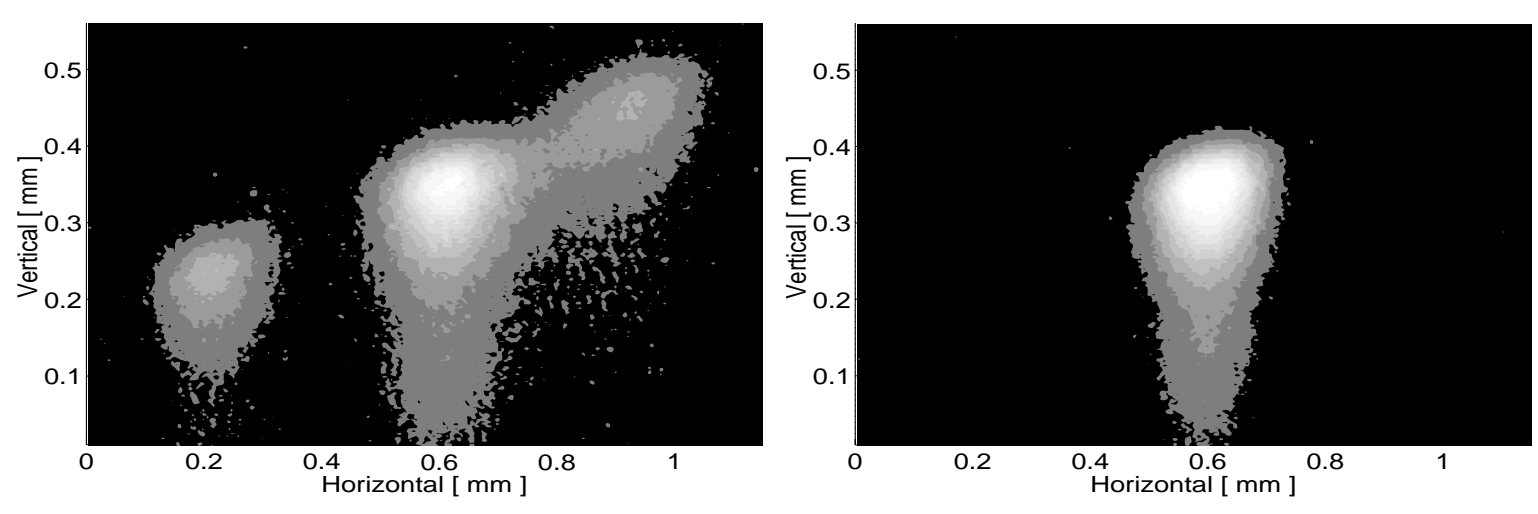

Figure 5: Synchrotron radiation image of the beam near the $3 \nu_{x}$ resonance. Left is the situation before the optic is corrected and right is the situation after the optic is corrected. (There is a distortion in the light optic in the vertical plane that is responsible for the vertical asymmetry of the image.

19\%. Knowing the errors in the machine we then adjusted quadrupoles in the machine to restore the lattice periodicity. After the quadrupoles were adjusted, beating of the $\beta$-function was reduced to less than $1 \%$.

We find that by restoring the lattice periodicity there have been several beneficial effects on the machine performance. The excitation of unallowed nonlinear resonances is suppressed as measured in the beam profile and beam tails. This suppression of resonances improves the injection efficiency and beam lifetime. As a result we regularly monitor the distortion of the periodicity and correct the optic when the $\beta$-beating becomes larger than $3 \%$. In addition, frequency map measurements of the ALS show that a well calibrated linear lattice can accurately describe the nonlinear behavior of the electrons in the storage ring.

\section{ACKNOWLEDGEMENTS}

The authors would like to thank the staff at the ALS, particularly Alan Jackson for his encouragement and support of the work and with whom we had many interesting discussions. Also we would like to thank Alan for making high precision power supply measurements. We wish to thank Gary Krebs for setting up the radiation monitors used in the tunescan experiments. We wish to thank Greg Portmann for helping to automate the tunescan measurements. Also we wish to thank Greg, Jim Hinkson and Jim Johnson for reducing the noise levels of the BPMs. We would like to thank Sasha Zholents for suggesting the tunescan technique for measuring resonance excitation. We wish to thank Sam Krinsky at BNL for encouraging one of the authors (J.S.) to participate in this work. We would also like to thank Martin Lee and Jeff Corbett at SLAC for interesting discussions.

\section{REFERENCES}

[1] W. J. Corbett, M. Lee, and V. Ziemann, in Proceedings of the 1993 Particle Accelerator Conference, Washington, p. 108.
[2] J. Safranek, in Proceedings of the 1995 Particle Accelerator Conference, Dallas, p. 2817.

[3] J. Safranek, Nucl. Inst. and Meth. A388, 27 (1997).

[4] J. Bengtsson and M. Meddahi, in Proceedings of the 1994 Particle Accelerator Conference, Berlin, p. 1021.

[5] D. Robin, J. Safranek, G. Portmann, and H. Nishimura, Proceedings of the 1996 Particle Accelerator Conference, Barcelona, p. 971.

[6] D. Robin, J. Safranek, and W. Decking, Phys. Rev. STAB, Vol. 2, 044001 (1999).

[7] C. Steier and D. Robin, Fully Coupled Analysis of Orbit REsponse Matrices at the ALS, these proceedings (2000).

[8] M. P. Woodley et al., Stanford Linear Accelerator Conference Report No. SLAC/PUB 3086, 1983, (unpublished).

[9] Written by H. Nishimura, J. Bengtsson and E. Forest

[10] Measurements made by Alan Jackson.

[11] A. B. Temnykh, in Proceedings of the IX ALL - Union Meeting on Accelerators of Charged Particles, Dubna, 1984, v. 2, p. 163.

[12] D. Robin et al., Proceedings of the 1995 Particle Accelerator Conference, Dallas, p. 2789.

[13] D. Robin, C. Steier, J. Laskar, and L. Nadolski, “Global Dynamics of the Advanced Light Source Revealed through Experimental Frequency Map Analysis”, accepted for publication in Phys. Rev. Lett.

[14] C. Steier, D. Robin, J. Laskar, and L. Nadolski, "Lattice Model Calibration and Frequency Map Measurements at the $A L S$ ", these proceedings. 